\title{
Fortbildungs- und andere Pflichten des «selbständigen» Arztes
}

\section{Brian Martina, Kathrin Agosti ${ }^{b}$, Rudolf Hauric, Giorgio Merlanid, Anna Cerutti-Marchesi ${ }^{e}$, Klaus Grätz ${ }^{f}$, Hanspeter Kuhn ${ }^{\mathrm{g}}$, Christoph Hänggeli ${ }^{\mathrm{h}}$, Jacques-André Romand ${ }^{\mathrm{i}}$}

a PD Dr. med., Kantonsarzt, Geschäftsfeld Medizin, Gesundheitsdirektion Zürich, Institut für Epidemiologie, Biostatistik und Prävention der Universität Zürich; b lic. iur., Geschäftsfeld Medizin, Gesundheitsdirektion Zürich; ' Dr. med., Kantonsarzt, Amtsleiter Amt für Gesundheit Zug, Präsident der Vereinigung der Kantonsärztinnen und Kantonsärzte der Schweiz VKS; ${ }^{d}$ Kantonsarzt, Medico cantonale, Dipartimento della sanità e della socialità, ufficio del medico

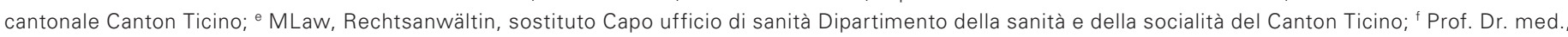

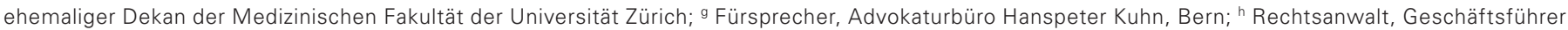
des Schweizerischen Instituts für ärztliche Weiter- und Fortbildung SIWF; ' Prof. Dr. med., médecin cantonal, service du médecin cantonal de la République et du canton de Genève, past président du Conseil de la Faculté de Médecine de I'Université de Genève

Der Erwerb und die Weiterentwicklung der ärztlichen Kenntnisse und Fertigkeiten erfolgen lebenslang in der Ausbildung bis zum Arztdiplom, über die Weiterbildung bis zum Facharzttitel sowie anschliessend in der Fortbildung. Besonders für die fachlich eigenverantwortliche Berufsausübung als Ärztin und Arzt sind zusätzlich Kenntnisse über die rechtlichen Rahmenbedingungen und Berufspflichten notwendig.

\section{Résumé}

L'acquisition et le développement des connaissances et compétences médicales s'effectuent tout au long de la vie, de la formation en vue de l'obtention du diplôme de médecin jusqu'à la formation continue, en passant par la formation postgraduée pour le titre de spécialiste. En raison de ses exigences élevées et des grandes responsabilités qu'elle implique, la profession de médecin est réglementée par la loi. Autrement dit, les médecins doivent également connaître les bases du cadre juridique de leur activité. En Suisse, le système de santé relève en principe des cantons, mais la Confédération, les cantons et les organisations médicales privées travaillent en fait de concert. Alors qu'un diplôme de médecin obtenu ou reconnu en Suisse autorise son titulaire à exercer sa profession sous surveillance professionnelle, un titre de spécialiste permet de pratiquer sous sa propre responsabilité. Les conditions requises pour l'obtention de l'autorisation d'exercer correspondante et les obligations professionnelles qui en découlent sont régies par la Loi fédérale sur les professions médicales. Les autorités sanitaires des cantons les contrôlent en tenant également compte des spécificités du droit cantonal. Les médecins disposent aussi de moyens constitutionnels de défense face aux décisions des autorités de surveillance. Elles peuvent par exemple être contestées auprès de l'autorité de recours compétente. Si le mécontentement porte sur les dispositions légales elles-mêmes, il convient alors de modifier ladite réglementation par la voie politique.
Der Arztberuf ist aufgrund seiner hohen Anforderungen und wegen der grossen Verantwortung staatlich reglementiert, ähnlich wie andere Gesundheitsfachberufe und beispielsweise auch der Anwaltsberuf. Dies bedeutet, dass Ärztin und Arzt auch Grundkenntnisse über die rechtlichen Rahmenbedingungen ihrer beruflichen Tätigkeit benötigen, besonders wenn sie diese fachlich eigenverantwortlich ausüben. Dafür ist eine kantonale Berufsausübungsbewilligung erforderlich. Einen umfassenden Überblick gibt der von der Schweizerischen Akademie der Medizinischen Wissenschaften SAMW und der FMH 2013 in überarbeiteter Fassung herausgegebene Leitfaden «Rechtliche Grundlagen im medizinischen Alltag» [1, 2]; die Implikationen der 2018 in Kraft getretenen Änderungen des Medizinalberufegesetzes sind in der Schweizerischen Ärztezeitung beschrieben worden [3]. Im vorliegenden Artikel sollen die wichtigsten Punkte für Ärztin und Arzt in der Praxis zusammengefasst werden.

Das Gesundheitswesen ist in der Schweiz grundsätzlich Sache der Kantone. Wie in vielen anderen Bereichen arbeiten aber auch hier Bund, Kantone und die privaten ärztlichen Organisationen zusammen.

Die angehende Humanmedizinerin beginnt ihre Laufbahn mit ihrer Ausbildung an den medizinischen 
Fakultäten der kantonal geführten Universitäten, neuerdings auch der Eidgenössischen Technischen Hochschule ETH. Die Bachelor- und Masterprüfungen werden von den Fakultäten durchgeführt, das Bundesamt für Gesundheit BAG ist zuständig für die Durchführung der Eidgenössischen Prüfung, Nachfolgerin des früheren "Staatsexamens». Die Eidgenössische Prüfung ist die Grundlage für die Verleihung des Arztdiploms durch das Eidgenössische Departement des Inneren EDI.

Nach Erwerb des Facharzttitels sind alle berufstätigen Ärztinnen und Ärzte zur lebenslangen Fortbildung verpflichtet.

Die Organisation der ärztlichen Weiterbildung erfolgt durch das Schweizerische Institut für Weiter- und Fortbildung SIWF als selbständiges Organ der FMH in Zusammenarbeit mit den medizinischen Fachgesellschaften. Das SIWF und seine 46 Weiterbildungsprogramme sind vom EDI 2018 erneut für weitere 7 Jahre akkreditiert worden. Das SIWF ist damit unverändert die Institution, welche in der Schweiz die eidgenössischen Facharzttitel erteilt, die gemeinsam mit dem EDI unterschrieben werden. Die Anerkennung von ausländischen Arztdiplomen und Facharzttiteln obliegt der Medizinalberufekommission MEBEKO als ausserparlamentarischer Kommission des Bundes, die administrativ dem BAG zugeordnet ist. Die MEBEKO ist seit 2018 auch für die Registrierung nicht anerkennbarer Diplome zuständig.

Nach Erwerb des Facharzttitels sind alle berufstätigen Ärztinnen und Ärzte zur lebenslangen Fortbildung verpflichtet. Die ärztliche Fortbildungspflicht zählt zu den Berufspflichten und ist auf Bundesebene festgeschrieben. Umfang und Modalitäten der Fortbildung werden vom SIWF zusammen mit den 45 Fachgesellschaften festgelegt und mit einem Fortbildungsdiplom bestätigt. Dieses kann über die Fortbildungsplattform des SIWF online beantragt werden.

\section{Umfang und Modalitäten der Fortbildung werden vom SIWF zusammen mit den 45 Fach- gesellschaften festgelegt und mit einem Fort- bildungsdiplom bestätigt.}

Die Kontrolle über die Erfüllung der Fortbildungspflicht erfolgt durch die zuständigen kantonalen Behörden.

Doktortitel und andere akademische Qualifikationen werden von den Universitäten vergeben. Ihre Verwendung wird von den Gesundheitsbehörden der Kantone überwacht, die sich ihrerseits in der Regel an den entsprechenden Empfehlungen von FMH und SIWF [4] orientieren.

\section{Von der «selbständigen» zur fachlich eigenverantwortlichen Berufsausübung}

Während ein Arztdiplom, das in der Schweiz erworben oder anerkannt wird, grundsätzlich zur Berufsausübung unter fachlicher Aufsicht berechtigt, zum Beispiel als Assistenzärztin in einer Weiterbildungsstätte, ist das Facharztdiplom der Zugang zur privatwirtschaftlichen Berufsausübung in eigener fachlicher Verantwortung. Der frühere Begriff der «selbständigen» Berufsausübung wurde aufgegeben, weil die fachliche Eigenverantwortung unabhängig zum Beispiel von einem Anstellungsverhältnis definiert wird. Nahezu alle Kantone kennen eine Bewilligungspflicht auch für die öffentlich-rechtliche Berufsausübung in eigener fachlicher Verantwortung, zum Beispiel für die ärztliche Leitung von Spitälern. Eine entsprechende Gesetzesrevision ist auch auf Bundesebene im Gange. Grundsätzlich ist die fachlich eigenverantwortliche Berufsausübung im Medizinalberufegesetz (Bundesgesetz über die universitären Medizinalberufe, MedBG) geregelt. Zulassung und Aufsicht erfolgen aber durch die Kantone, die dabei auch zusätzliche kantonale Gesetzesgrundlagen berücksichtigen. Tabelle 1 zeigt beispielhaft für die Kantone Genf, Tessin, Zug und Zürich die zuständigen Institutionen und die wichtigsten zusätzlichen kantonalen Gesetzesgrundlagen.

Im Medizinalberufegesetz sind die fachlichen und persönlichen Voraussetzungen für eine Berufsausübungsbewilligung definiert: eidgenössisches oder anerkanntes Arztdiplom und entsprechender Weiterbildungstitel, Vertrauenswürdigkeit, physische und psychische Gewähr für eine einwandfreie Berufsausübung sowie das Beherrschen der Amtssprache des jeweiligen Kantons. Zudem ist im Bundesgesetz auch festgelegt, welche Berufspflichten eingehalten werden müssen: sorgfältige und gewissenhafte Berufsausübung, lebenslange Fortbildung, Wahrung der Patientenrechte, weder irreführende noch aufdringliche Werbung, Handeln unabhängig von finanziellen Vorteilen, Wahrung des Berufsgeheimnisses, Beistand in dringenden Fällen, Mitwirkung im Notfalldienst nach kantonaler Vorgabe, Abschluss einer Berufshaftpflichtversicherung entsprechend den Risiken der Tätigkeit. Dazu kommen Besonderheiten des kantonalen Rechts, beispielsweise Befristungen der Bewilligungen.

Die Überprüfung von Voraussetzungen und Berufspflichten unterscheidet sich aufgrund dieser Situation von Kanton zu Kanton. Ein Fehlen der Voraussetzun- 
Tabelle 1: Wichtigste Gesetzesgrundlagen und zuständige Behörden für Zulassung und Aufsicht der ärztlichen Berufsausübung inklusive aufsichtsrechtlicher Rechtsmittelinstanzen oder vereinfacht «Rekursinstanzen», illustriert am Beispiel der Kantone Genf, Tessin, Zug und Zürich. Arztdiplome, Weiterbildungsdiplome oder Facharzttitel und akademische Qualifikationen wie Doktortitel werden in der Regel lebenslang verliehen. Kantonale Berufsausübungsbewilligungen sind abhängig von entsprechenden Voraussetzungen und der Erfüllung der Berufspflichten.

\begin{tabular}{|c|c|c|c|c|}
\hline & Kanton Genf & Kanton Tessin & Kanton Zug & Kanton Zürich \\
\hline Zweite «Rekursinstanz» & $\begin{array}{l}\text { Chambre administrative } \\
\text { (Cour de droit public } \\
\text { de la Cour de Justice) }\end{array}$ & $\begin{array}{l}\text { Tribunale Cantonale } \\
\text { Amministrativo }\end{array}$ & $\begin{array}{l}\text { Kantonales Verwaltungs- } \\
\text { gericht }\end{array}$ & $\begin{array}{l}\text { Kantonales Verwaltungs- } \\
\text { gericht }\end{array}$ \\
\hline Erste «Rekursinstanz» & - & $\begin{array}{l}\text { Servizio ricorsi del Consiglio } \\
\text { di Stato del Canton Ticino }\end{array}$ & Regierungsrat & $\begin{array}{l}\text { Geschäftsfeld Dienstleistun- } \\
\text { gen der Gesundheitsdirektion, } \\
\text { Abteilung Rechtsmittel }\end{array}$ \\
\hline $\begin{array}{l}\text { Kantonale Aufsichts- } \\
\text { behörde }\end{array}$ & $\begin{array}{l}\text { Direction générale de la santé } \\
\text { - démarches administratives, } \\
\text { service du médecin cantonal }\end{array}$ & $\begin{array}{l}\text { Medico cantonale } \\
\text { (vom Dipartimento della } \\
\text { sanità e della socialità dele- } \\
\text { gierte Zuständigkeit) }\end{array}$ & $\begin{array}{l}\text { Kantonsarzt (von der } \\
\text { Gesundheitsdirektion } \\
\text { delegierte Zuständigkeit) }\end{array}$ & $\begin{array}{l}\text { Geschäftsfeld Medizin der } \\
\text { Gesundheitsdirektion, Abtei- } \\
\text { lung Gesundheitsberufe und } \\
\text { Bewilligungen }\end{array}$ \\
\hline $\begin{array}{l}\text { Kantonale Bewilligungs- } \\
\text { behörde }\end{array}$ & $\begin{array}{l}\text { Direction générale de la santé } \\
\text { - démarches administratives, } \\
\text { service du médecin cantonal }\end{array}$ & $\begin{array}{l}\text { Ufficio di sanità (vom } \\
\text { Dipartimento della sanità } \\
\text { e della socialità delegierte } \\
\text { Zuständigkeit) }\end{array}$ & $\begin{array}{l}\text { Kantonsarzt (von der } \\
\text { Gesundheitsdirektion } \\
\text { delegierte Zuständigkeit) }\end{array}$ & $\begin{array}{l}\text { Geschäftsfeld Medizin der } \\
\text { Gesundheitsdirektion, } \\
\text { Abteilung Gesundheitsberufe } \\
\text { und Bewilligungen }\end{array}$ \\
\hline \multirow[t]{2}{*}{$\begin{array}{l}\text { Wichtigste Gesetzes- } \\
\text { grundlagen }\end{array}$} & $\begin{array}{l}\text { Loi cantonal sur la santé du } \\
7 \text { avril } 2006 \text { (LS; K } 1 \text { 03) }\end{array}$ & $\begin{array}{l}\text { Legge sulla promozione della } \\
\text { salute e il coordinamento } \\
\text { sanitario (LSan) }\end{array}$ & $\begin{array}{l}\text { Kantonales Gesundheits- } \\
\text { gesetz GesG }\end{array}$ & $\begin{array}{l}\text { Kantonales Gesundheitsgesetz } \\
\text { GesG }\end{array}$ \\
\hline & $\begin{array}{l}\text { Medizinalberufegesetz des } \\
\text { Bundes MedGB }\end{array}$ & $\begin{array}{l}\text { Medizinalberufegesetz } \\
\text { des Bundes MedGB }\end{array}$ & $\begin{array}{l}\text { Medizinalberufegesetz } \\
\text { des Bundes MedGB }\end{array}$ & $\begin{array}{l}\text { Medizinalberufegesetz } \\
\text { des Bundes MedGB }\end{array}$ \\
\hline
\end{tabular}

gen führt aber zu einer Ablehnung des Antrags auf eine Berufsausübungsbewilligung; das Wegfallen der Voraussetzungen führt zu deren Entzug oder Erlöschen. Eine Verletzung der Berufspflichten führt nicht automatisch $\mathrm{zu}$ einem Verlust der Bewilligung, sie kann je nach Schwere disziplinarische Massnahmen unterschiedlichen Ausmasses nach sich ziehen, allenfalls auch Grund für eine strafrechtliche Anzeige sein. So kann zum Beispiel eine isolierte Verletzung der Fortbildungspflicht lediglich einen Verweis oder eine Busse zur Folge haben.

Die Berufsausübungsbewilligung berechtigt zur fachlich eigenverantwortlichen Tätigkeit als Ärztin und Arzt, also zum Beispiel zum berufsmässigen Führen einer eigenen Praxis und zum Ausstellen von ärztlichen Rezepten. Geregelt sind diese Tätigkeiten in den entsprechenden Bundesgesetzen - also zum Beispiel im

\section{Die Berufsausübungsbewilligung berechtigt zur fachlich eigenverantwortlichen Tätigkeit als Ärztin und Arzt.}

Medizinalberufe-, Heilmittel- und Betäubungsmittelgesetz - sowie kantonalen Gesetzen und Verordnungen. Nicht jede ärztliche Tätigkeit ist aber abhängig von einer Berufsausübungsbewilligung. So ist zum Beispiel die ärztliche Beratung und Betreuung im privaten Rahmen ohne eine solche möglich, und auch die allgemeine Nothilfepflicht nach Stand der individuellen Kenntnisse und Fertigkeiten besteht unabhängig von irgendeinem Bewilligungsstatus. Zudem gibt es im Medizinalberufegesetz nicht nur aufsichtsrechtliche, sondern auch strafrechtliche Bestimmungen zur unberechtigten Verwendung von medizinischen Qualifikationen und zur Beschäftigung von Ärzten, die nicht im entsprechenden Register eingetragen sind.

\section{Zunehmende Regulierung und Trans- parenz, aber auch rechtsstaatliche Möglichkeiten für Betroffene}

Das Wachstum des Gesundheitsmarkts, die zunehmende Mobilität der Ärzteschaft, aber auch die Beschleunigung des medizinischen Fortschritts haben dazu geführt, dass die Kontrolle im Interesse der $\mathrm{Pa}$ tientensicherheit nur über zunehmende Formalisierung, Regulierung und Kontrolle sichergestellt werden kann. Dabei stellen die gesetzlichen Regelungen grundsätzlich rechtlich verbindliche Minimalkriterien dar; fachlich begründet gibt es oft weitergehende Standards, seien dies Empfehlungen von Fachgesellschaften oder standesrechtliche Normen.

Gleichzeitig wird auch dem gestiegenen Informationsbedürfnis einer kritischen Öffentlichkeit Rechnung getragen. Im Medizinalberuferegister MedReg (www. medregom.admin.ch) sind heute schon Arztdiplom, Weiterbildungstitel und Bewilligungen zur fachlich eigenverantwortlichen Berufsausübung öffentlich einsehbar, im Ärzteverzeichnis der FMH (www.doctorfmh. ch) zusätzlich auch der aktuelle Fortbildungsstatus. 
Wenn ein Arzt aus Unwissenheit zum Beispiel ein Gesuch um eine Berufsausübungsbewilligung einreicht, ohne über einen Facharzttitel oder den Weiterbildungstitel «Praktischer Arzt» zu verfügen, so wird er in der Regel von der Behörde darüber informiert, dass er die entsprechenden Voraussetzungen nicht erfüllt, und es wird ihm Gelegenheit gegeben, seinen Antrag zurückzuziehen. Ärztinnen und Ärzte haben aber natürlich auch rechtsstaatliche Möglichkeiten, sich gegen Entscheide der Aufsichtsbehörden zu wehren. So kann ein Antragsteller eine Verfügung verlangen und diese bei der entsprechenden Rechtsmittelinstanz (vereinfacht "Rekursinstanz», siehe Tab. 1) anfechten. Die kantonalen Behörden berücksichtigen bei ihrer Umsetzungspraxis neben den gesetzlichen Grundlagen auch Entscheide der Gerichte zu früheren Rekursfällen. Die Besonderheiten eines aufsichtsrechtlichen Verfahrens sind in Tabelle 2 aufgeführt.

Falls die Unzufriedenheit sich gegen die gesetzlichen Grundlagen richtet, stehen die politischen Möglichkeiten auf den entsprechenden Ebenen zur Verfügung. Hier kommt den ärztlichen Berufsverbänden eine wichtige Rolle zu, denn zu ihren Aufgaben gehört es, bei gesetzgeberischen Prozessen die Interessen der Ärzteschaft einzubringen.

Korrespondenz: PD Dr. med. Brian Martin, MPH

Facharzt für Prävention und Gesundheitswesen Gesundheitsdirektion ZH Kantonsarzt

Stampfenbachstrasse 30

Postfach

CH-8090 Zürich

Tel. 0432592409

Fax 0432595151

kantonsarzt.sekretariat[at]

gd.zh.ch
Die grosse Mehrzahl aller bewilligungs- und aufsichtsrechtlichen Fragen wird aber ohne abschlägige Verfügungen oder gar richterliche Entscheidungen gelöst. Die kantonalen Gesundheitsbehörden stehen der Ärzteschaft für Auskünfte zur Verfügung, ebenso wie die Ärztegesellschaften.

\section{Interessenverbindungen}

Alle Interessenverbindungen ergeben sich aus den Affiliationen in der Autorenliste.
Tabelle 2: Besonderheiten eines aufsichtsrechtlichen Verfahrens.

Adressaten eines aufsichtsrechtlichen Verfahrens der kantonalen Gesundheitsbehörden sind in aller Regel Bewilligungsinhaberinnen und Bewilligungsinhaber, in Ausnahmefällen auch andere Gesundheitsfachpersonen.

Bewilligungsinhaberinnen und Bewilligungsinhaber sind zur Mitwirkung in aufsichtsrechtlichen Verfahren verpflichtet.

Meldungen können durch die Bevölkerung, andere Gesundheitsfachpersonen oder Behörden erfolgen. Meldende Personen werden nicht zur Partei in einem allfälligen Verfahren und dürfen in der Regel nicht über den weiteren Verlauf informiert werden.

Aufsichtsrechtliche Verfahren beschäftigen sich nur mit medizinischen Behandlungen, sofern Implikationen bezüglich Bewilligungsvoraussetzungen oder Berufspflichten zu erwarten sind.

Aufsichtsrechtliche Verfahren unterliegen dem Amtsgeheimnis. Nur in Ausnahmefällen wird das öffentliche Interesse höher gewichtet und entsprechend informiert.

Das Medizinalberuferecht des Bundes regelt, welche Informationen der Öffentlichkeit im Medizinalberuferegister MedReg zugänglich gemacht werden.

Gesundheitsfachpersonen, die Adressaten eines aufsichtsrechtlichen Verfahrens werden, können einen Rechtsvertrete oder andere Vertrauenspersonen zuziehen, um ihre Rechte wahrzunehmen.

Literatur

1 SAMW, FMH (Hrsg.). Rechtliche Grundlagen im medizinischen Alltag. Ein Leitfaden für die Praxis. 2. Auflage. Muttenz: Schwabe; 2013.

2 Pally U, Salathé M. Rechtliche Grundlagen im medizinischen Alltag. Schweiz Ärzteztg. 2013;94(17):645

3 Hänggeli C, Kuhn H. Das Medizinalberufegesetz (MedBG) tritt am 1.1.2018 in Kraft. Schweiz Ärzteztg. 2017;98(51-52):1727-8.

4 Hänggeli C, Kuhn H, Linder B. Titelausschreibung leicht gemacht. Schweiz Ärzteztg. 2015;96(50-51):1834-6. 